\title{
Let me Introduce Myself: I am Care-O-bot 4, a Gentleman Robot
}

\author{
Ralf Kittmann ${ }^{1}$, Tim Fröhlich², Johannes Schäfer ${ }^{1}$, Ulrich Reiser ${ }^{2}$, Florian \\ Weißhardt ${ }^{2}$, Andreas Haug ${ }^{1}$ \\ Phoenix Design $\mathrm{GmbH}+\mathrm{Co} . \mathrm{KG}^{1}$ \\ Fraunhofer Institute for Manufacturing Engineering and Automation ${ }^{2}$
}

\begin{abstract}
In this paper, we present the latest step in the evolution of the Care-O-bot: A general purpose service robot that assists users in various tasks where human-like behavior and interfaces are desired. We argue that technology, design and user experience have to match to create a robot that will blend not only visually, but also emotionally into the scenarios it will be used for. We describe the requirement priorities, design decisions and technical feasibility with which Care-O-bot 4 is able to fulfill tasks in human environments and additionally evokes positive emotions and thus furthering and enhancing human-robot interaction (HRI).
\end{abstract}

\section{Introduction}

The first impression matters. In human interaction this holds true, in human-robot interaction it may make the difference between curiosity and sympathy vs. fear and rejection.

Care-O-bot 4 was designed as a gentleman robot that allows the users to relate emotionally and socially with it. It is the result from an evolution of robots from 1998 till 2015 - from Care-O-bot I (Schaeffer 1999) and II (Graf 2004) to Care-O-bot 3 (Reiser et al. 2009), see Figure 1.

Care-O-bot 4 does not only address technical research groups by providing a reliable platform, it also aims at the need of research in the fields of human-robot-interaction and social behavior. One of the key advancements of Care-O-bot 4 is its modular approach that enables a cost efficient, distributed and customized research. Other improvements were made in a higher agility (e.g. the spherical joints), options for multi-modal interactivity (integrated in neck and head) and better presence.

In this paper, we'll show the emotional approach to the design of the Care-O-bot 4: First, we show related work and how the Care-O-bot evolved during years of research. Then, we'll give 
a theoretical framework for emotional design and show how it translates into concrete design choices and influenced the technical implementation. We'll conclude with a summary and outlook.

\section{Related Work}

\subsection{Recent Advances in the Field of Service Robotics}

During the past years, the service robotics research community and markets have been developing at an increasing speed (IFR Statistical Department 2015). For simple, non-manipulating service robots commercial solutions in hotels, hospitals and retail stores are evolving, e.g. Oshbots (NASA Fellow Robots 2015), Savioke (CREATIVA77, 2015), RoboCourier (Swisslog AG 2015). More complex service robots with manipulation capabilities are still restricted to the use as research platforms, e.g. TiagoBot (PAL Robotics SL 2015), RB1 (Ackermann 2015), $P R 2$, iCub (Metta 2008). Usually, these robots are designed for a certain research domain. Platforms for research in grasping manipulation usually do not offer a lot of interaction possibilities. Research robots dedicated to HRI usually constrain to the hardware components providing the interaction modalities and do not encompass the full embodiment for realworld applications, e.g. Simon (Georgia Institute of Technology 2012), Flobi (Lütkebohle 2010).

Care-O-bot is outstanding in that respect: It offers the embodiment for both mobile manipulation and human-robot interaction. Thus, it is suited very well for real-world experiments involving real users.
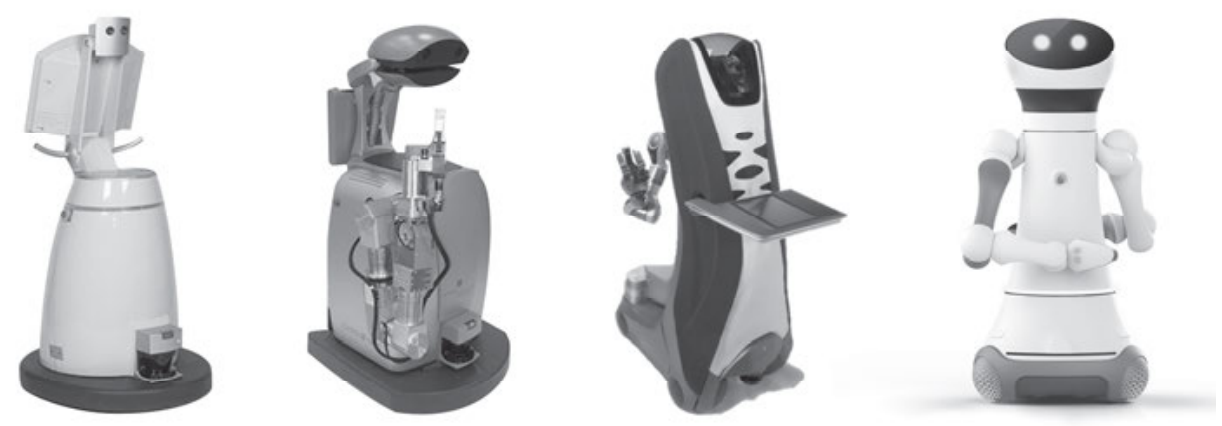

Figure 1: Evolution of Care-o-Bot, from technological feasibility to acceptable embodiment and Care-O-bot 4. 


\subsection{The Care-O-bot Project}

In the first two Care-O-bot generations, the development of service robotics was focused on these key components: navigation, manipulation and perception. Care-O-bot 3 in contrast was already designed as a product vision for a future household assistant. The capabilities and the intended social roles of Care-O-bot 3 were reflected in an iconic design for a mobile service robot delivering fetch and carry services (Parlitz 2008).

Key design elements are

- an abstract anthropomorphic appearance (discreet service of a butler),

- the 'two-sides' concept referring to a user-oriented side for HRI and a working side with the arm that is located at the back of the robot,

- animation and metamorphosis: covers consisting of soft and hard materials for a body gesturing torso and a comfortable haptics.

Although there is an agreement that too anthropomorphic appearance bears the danger of raising too high expectations regarding its capabilities or rejection, the exact degree of humanlikeliness is still a topic of research in the robotics community. The uncanny valley even predicts fearful reactions for almost human-like machines (Mori, MacDorman \& Kageki 2012).

Meyer (2011) published a study on the acceptance of robots to assist in their daily lives among senior citizens. It was found that robots are not expected to look like humans, but they should behave like humans to get easier accepted. It is important, that the users get feedback on the inner state of the robot to be able to estimate its presumable next actions. According to Tapus and Mataric (2006), social robots should have emotional expression ability. This capability helps to create empathy and thus establish a link between user and robot. Riek et al. (2009) observe that people are more empathetic toward human-like robots and less empathetic toward mechanical-looking robots.

Thus, the degree of human-likeliness was considered carefully for Care-O-bot 4. Although the principle of the abstract human appearance was to be sustained, Care-O-bot 4 should be able to create empathy. It should have an emotional appeal and be able to provide multi-modal intuitive feedback on its inner state and understanding of the context.

\section{Emotional Design}

To achieve the goal of an attractive and approachable robot, it was developed in a multi disciplinary team. This team included product designer, robotics and software engineers on the one hand, product, interaction and UX designers on the other. This project team worked closely together as proposed by the human-centered design process (DIN EN ISO 9241-210, 2010). Requirement priorities, design decisions and technical feasibility were set, discussed and carefully balanced in the team. 
The human emotional reaction to 'everyday things' can be described along three levels (Norman 2004, 2013): Visceral, behavioral and reflective. The first being about 'appearance', the second describing 'pleasure and effectiveness of use' and the last relating to 'self-image, personal satisfaction, memories'.

To build up a lasting emotional response, Care-O-bot 4 needs to appeal on all three levels. The robot has been designed to evoke an immediate positive emotional response on the visceral level. Its construction includes all the abilities to create positive interactions on the behavioral level. To excel at the reflective level as a robot, it needs to integrate socially, too.

As a 'social actor' the robot conveys social cues, especially at a physical level, i.e. body and movement as well as face, eyes; other cues are language, social dynamics, social roles and psychological cues (Fogg 2002; as cited in Norman 2004). The Care-O-bot 4 covers the physical level by hardware design and is equipped to relate on language, social dynamics and social roles (the psychological level is hard to attain: it involves personality, empathy and humor).

The body involving posture and expressive movements is closely related to the first impression and thus to the visceral reaction. It also transmits important cues on the physical level. The other important physical cues are face and eyes and closely related with these the behavioral interactions that ensue between the user and the robot. The next two sections show how we approached these aspects of emotional design.

\subsection{Body, Posture and Expressivity}

Care-O-bot 4 as a general purpose robot should to be immediately appealing at first glance on the visceral level to a broad range of persons: It is designed to transmit the image of a 'Gentleman' (Figure 2). A gentleman is always present without being obtrusive, offering help and assistance in a courteous and independent way.

The design is very figurative (Figure 4): A closed silhouette and sculptural slender appearance with two arms and a small symmetrical physical footprint. This figure is meant to resemble some human features without being itself humanoid.
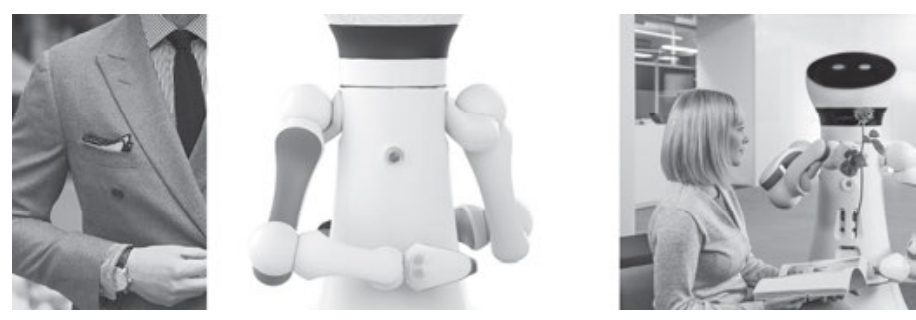

Figure 2: First impression of a 'Gentleman' and typical body language (both for a human and Care-O-bot 4); natural and expressive movements in social situations reinforce this attitude

To achieve high expressivity, the Care-O-bot 4 should be able to move in a human-like way. This concerns posture and natural movements of the arms. 
The body itself is divided into three parts, similar to the human body: 'head', 'trunk' and 'base' are joined at the pivotal points 'neck' and 'hip' in a natural way, i.e. they give agility similar to human movements. Thus Care-O-bot 4 sends physical cues that are familiar and sociable, e.g. leaning forward in kind of a 'bow' without losing balance or eye contact (Figure 3).
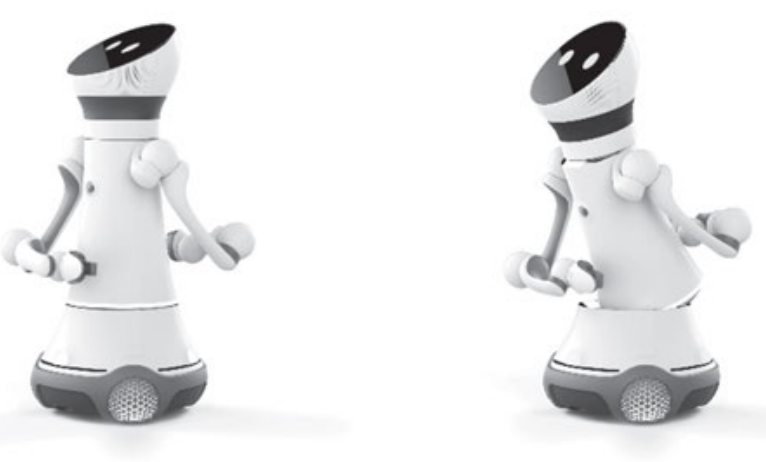

Figure 3: Natural posture and movements sending familiar and sociable physical cues

Exposing these joints and movements to be seen and understood by the user makes the robot more human-like and predictable. It allows to quickly build a consistent and adequate mental model which is the basis for good usability (Norman 2013, DIN EN ISO 9241-110, 2006) and thus very closely related to 'effectiveness of use'.

Other features of the Care-O-bot 4 support this notion, too: The base shows that locomotion is obviously possible (on flat surfaces); the two arms are clearly able to grasp objects and interact with the environment and humans.

The arms were significantly changed from the Care-O-bot 3 since they were not well perceived by users. Care-O-bot 4 now may have two arms located analogous to human arms. The principle structure remained unchanged, but appearance was greatly improved: the new arms are slimmer, lighter and more approachable; they now clearly indicate a front and backside. This allows greater expressivity in the movements, e.g. to show more respect towards a human counterpart the robot should not expose the inner parts (Figure 2), or it could open the arms wide in an inviting gesture.

\subsection{Facial Expressions and Multi-Modal User Interface}

Another important feature is the 'head' of Care-O-bot 4: It may show facial expressions, a rich graphical user interface and contains input and output units for multi-modal interactions.

Facial expressions are important to express emotions and relate socially, e.g. EMYS (Kędzierski et al. 2013) or Kismet (Breazeal 2000). From a distance Care-O-bot 4 shows facial expressions on the screen embodied into the 'head' to make and stay in contact. We opted for a minimalistic pair of eyes which - supported by the movements of the head - are sufficient to 
express a wide variety of emotions, see e.g. the robot characters in the movie 'WALL-E' (The Walt Disney Company 2015) and Figure 4.
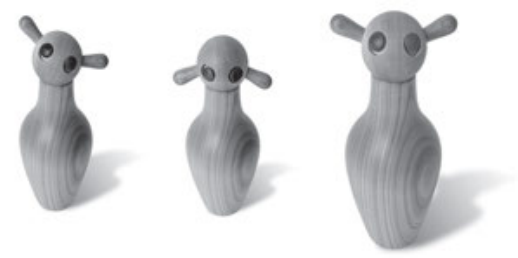

Figure 4: Simple figurine (wood puppet) that is able to express emotions only by simple eyes, head position and rotation, even without elaborate facial expressions (Berendsohn AG, 2015)

When the user approaches the Care-O-bot 4 the facial expression gives way to a rich graphical user interface appropriate for the situation. The 'head' then works as a touch screen which is easy to reach and interact with (Figure 7). We do expect (and informal user reactions agree) that "touching into the face" poses no serious constraints to the users of the robot due to its sculptural and less anthropomorphic design. Additionally, the upper part ('head' and neck) is equipped to enable a wide range of interactions: react to the user's gestures (cameras and sensors); natural language (microphones and speakers). Thus, Care-O-bot 4 offers a multi-modal user interface and a wide range of possibilities for rich, engaging and emotionally pleasing interactions with its users. It is now up to the given task and environment to define and implement a good user experience at this level.

\section{Technical Implementation}

In this section, we give a concise overview on the implementation of the technical, functional and design requirements. For the realization of the concepts, consistent modularity was an important guiding principle. All modules are easy to compose via a connector including power supply, safety circuits (including safety-torque-off) and communication lines (Ethernet, CAN). The individual modules possess their own computation unit, hence the integration of modules works according to the Plug'n'Play principle.

\subsection{Spherical Joints for Agility and Expressivity}

To achieve the goals set by both design and functional requirements, in particular the high agility and desired work space specification (reach both floor level and shelves up to 1.6 meters) had to be considered. The sculptural appearance introduced challenging constraints for construction space. Furthermore, statics had to be ensured for all robot configurations - in the worst case two stretched-out arms carrying the maximum payload. In order to achieve these goals in a very compact space, a new 3-degrees-of-freedom kinematics was developed. The rotational axes of the first and third joint are aligned, while the rotational axis of the second 
joint is translated and rotated such that all axes meet in one point, the effective center of motion (Figure 5). The link attached to the first axis can be moved on a sphere originating in the center of motion. The angle between the z-axes of first and second axes determine the maximum tilting angle of the kinematics. These constructions were used as pivot points for both 'hip' and 'neck' of Care-O-bot 4. Figure 5 (right) shows the implementation of the kinematics for the neck joint.
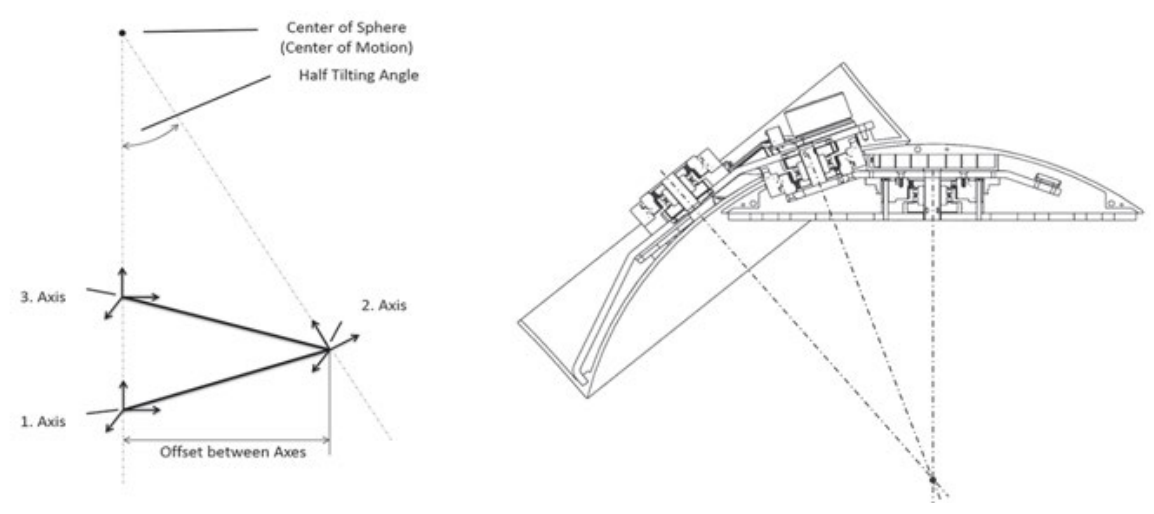

Figure 5: Kinematic principle (left) and implementation example of spherical joint (right)

\subsection{Head for Multi-Modal User-Interaction}

For intuitive communication with users, multi-modal interaction possibilities are foreseen in the head module. Regarding the communication direction user to robot, Care-O-bot 4 possesses a microphone for speech recognition and cameras for person and gesture recognition. For the opposite communication direction, Care-O-bot 4 offers speakers, LEDs to signal its inner state and a laser pointer in the hand e.g. to disambiguate objects to fetch. Care-O-bot 4's spherical joints can be used for body and head gestures such as bowing, nodding or shaking the head. Furthermore, the touch screen can be positioned appropriately depending on the height and situation of the user (sitting/standing) using hip and head joint.

First experiments to implement the oval display included rear-projection techniques using highly reflective films. Due to the lower contrast and resolution compared to current LEDbased screens and the small projection length available in the head, a touchscreen was chosen. 


\subsection{Modular Hands-Arm System}

The arms are composed of four self-contained motor units (three SCHUNK ${ }^{1}$ Powerball ERB modules and one PRL+ 100 module in the shoulder). The use of the Powerballs had two major advantages: the links between the balls could be almost freely designed in size and shape in order to achieve both the intended appearance and workspace of the arms.

An innovative one-finger hand shown in Figure 6 was developed with Schunk especially for Care-O-bot 4. It has two-degrees of freedom and a stiff palm. Integrated is furthermore a laser pointer, a 3D-camera to grasp objects in spaces not covered by the torso sensors and LEDs to light dark areas. It was

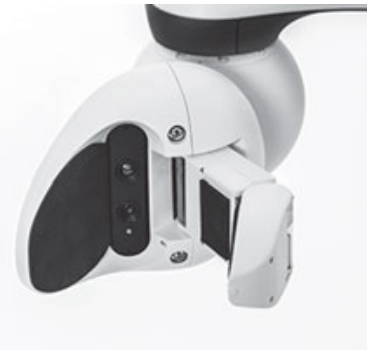

Figure 6: Hand of Care-O-bot 4 with $3 D$-camera, $R G B-L E D$ s and laser pointer designed such that it can operate typical household infrastructure (drawers, light switches, etc.) and manipulate various household objects from silverware to bottles, cans and boxes.

\section{Summary and Outlook}

Care-O-bot 4 is a general purpose service robot that is apt to adapt to different environments, situations and requirements. Its design blends in every configuration with the situation and it is able to create a positive emotional bond on all three levels (visceral, behavioral, and reflective) providing the necessary social cues. As with its predecessors, the Care-O-bot 4 may be deployed in different environments from a home setting to a medical assistant (Figure 7) and assumes different roles, where it is perceived as social actor (Heuwinkel 2013). With its appearance and rich possibilities for interaction designers, engineers and developers have a large set of tools to create and implement a compelling positive user experience for each environment and task. Care-O-bot 4 will be used in real-world applications to validate in particular the emotional design put forward in this paper. Beyond that, it enables a broadened scope to many research projects exploring user experience and emotions in the field of human-robot interac-
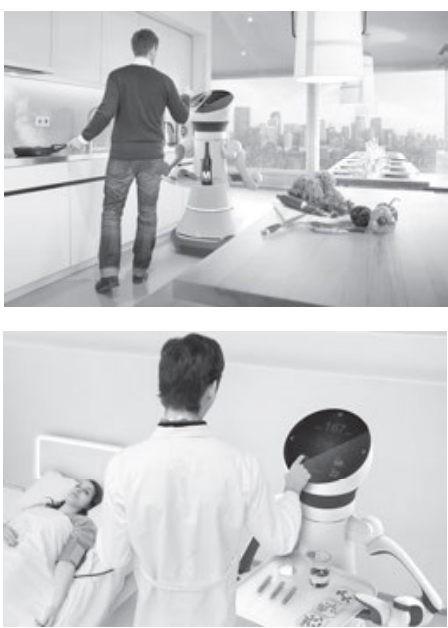

Figure 7: Care-O-bot 4 in different social situations. tions. 


\section{Kontaktinformationen}

Phoenix Design $\mathrm{GmbH}+\mathrm{Co} . \mathrm{KG}$

Kölner Str. 16, 70376 Stuttgart, www.phoenixdesign.com

Corresponding author: johannes.schaefer@phoenixdesign.com

Fraunhofer Institute for Manufacturing Engineering and Automation (Fh-IPA)

Postfach 800469, 70504 Stuttgart, http://www.ipa.fraunhofer.de/

Corresponding author: tim.froehlich@ipa.fraunhofer.de

\section{Bibliography}

Ackerman, E. (2015). Robotnik Enters Mobile Manipulator Market With RB-1. Retrieved 2015, March 30 from http://spectrum.ieee.org/automaton/robotics/industrial-robots/robotnik-enters-mobile-manipulator-market-with-the-rb1

Breazeal, C. (2000). Sociable Machines: Expressive Social Exchange between Humans and Robots. Doctoral dissertation, Massachusetts Institute of Technology, USA.

Berendsohn AG (2015). Blue Line Kollektion - Blфk, zeigen Sie Ihre Gefühle. Retrieved 2015, March 24 from http://www.berendsohn.de/blue-line/alfredo-haeberli

CREATIVA 77 (2015). Savioke. Retrieved 2015 March 30 from http://www.savioke.com/

DIN EN ISO 9241-110. (2006). Ergonomics of Human-System Interaction - Part 110: Dialogue Principles. Berlin: Beuth.

DIN EN ISO 9241-210. (2010). Ergonomics of Human-System Interaction - Part 210: Human-Centred Design for Interactive Systems. Berlin: Beuth.

Georgia Institute of Technology (2012). Socially Intelligent Machines Lab - Simon the Robot. Retrieved 2015 March 30 from http://www.cc.gatech.edu/social-machines/projects.html

Graf, B., Hans, M. \& Schraft, R.D. (2004). Care-O-bot II - Development of a Next Generation Robotic Home Assistant. Autonomous Robot Journal, 16(2), 193-205.

Heuwinkel, K. (2013). Framing the Invisible - The Social Background of Trust. In R. Trappl (Ed.): Your Virtual Butler. Heidelberg: Springer. [p. 16-26]

IFR Statistical Department (2015). World Robotics - Service Robots. Retrieved 2015, March 30 from www.worldrobotics.org

Kędzierski, J., Muszyński, R., Zoll, C., Oleksy, A. \& Frontkiewicz, M. (2013). EMYS—Emotive Head of a Social Robot. International Journal of Social Robotics, 5(2), 237-249.

Lütkebohle, I., Hegel, F., Schulz, S., Hackel, M., Wrede, B., Wachsmuth, S., \& Sagerer, G. (2010). The Bielefeld Anthropomorphic Robot Head "Flobi", 2010 IEEE International Conference on Robotics and Automation, http://aiweb.techfak.uni-bielefeld.de/pubs/2009/bielefeld-anthropomorphic-robothead-flobi

Metta, G., Sandini, G., Vernon, D., Natale, L., \& Nori, F. (2008). The iCub humanoid robot: an open platform for research in embodied cognition. In Proceedings of the 8th Workshop on Performance Metrics for Intelligent Systems (PerMIS '08). ACM, New York, USA. (p. 50-56). doi: $10.1145 / 1774674.1774683$ 
Meyer, S. (2011). Mein Freund der Roboter. Servicerobotik für ältere Menschen - eine Antwort auf den demographischen Wandel? Berlin: VDE.

Mori, M., MacDorman, K.F., Kageki, N. (2012). The Uncanny Valley. IEEE Robotics \& Automation Magazine, 19(2), 98-100.

NASA Research Park (2015). Fellow Robots. Retrieved 2015, March 30 from http://fellowrobots.com/robots

Norman, D. (2013). Design of Everyday Things: Revised and Expanded Edition. New York: Basic Books. First edition 1989, originally published as "Psychology of Everyday Things" (1988).

Norman, D. (2004). Emotional design: Why we love (or hate) everyday things. New York: Basic Books.

PAL Robotics SL (2015). Tiago Mobile Manipulator. Retrieved 2015, March 30 from http://tiago.palrobotics.com/en/

Parlitz, C., Hägele, M., Klein, P., Seifert, J. \& Dautenhahn, K. (2008). Care-O-bot 3 - Rationale for Human-Robot Interaction Design. In International Federation of Robotics, Robot Research Initiative $C N U$, Pohang Institute of Intelligent Robotics, Institute of Control, Robotics and Systems -ICROS (Ed.): 39th International Symposium on Robotics ISR 2008, Seoul, South Korea. (p. 275-280).

Reiser, U., Connette, C., Fischer, J., Kubacki, J., Bubeck, A., Weisshardt, F., Jacobs, T., Parlitz, C., Hägele, M. \& Verl, A. (2009). Care-O-bot 3 - Creating a Product Vision for Service Robot Applications by integrating Design and Technology. In: Institute of Electrical and Electronics Engineers \& Robotics Society of Japan (Ed.): International Conference on Intelligent Robots and Systems IROS 2009, St. Louis, USA. (p. 1992-1998).

Riek, L., Rabinowitch, T.C., Chakrabarti, B. \& Robinson, P. (2009). How anthropomorphism affects empathy toward robots. In Association for Computing Machinery \& IEEE Computer Society (Ed.): 4th International Conference on Human Robot Interaction HRI 2009, New York, USA. (p. 245-246). doi: $10.1145 / 1514095.1514158$.

Schaeffer, C. \& May, T. (1999). Care-O-bot: A System for Assisting Elderly or Disabled Persons in Home Environments. In Proceedings of AAATE-99, Düsseldorf.

Swisslog AG (2015). RoboCourier Mobiler Roboter. Retrieved 2015, March 30 from http://www.swisslog.com/de/Products/HCS/Automated-Material-Transport/RoboCourier-Autonomous-Mobile-Robot

Tapus, A. \& Mataric, M. (2006). Emulating Empathy in Socially Assistive Robotics. Retrieved 2015, March 25 from http://robotics.usc.edu/publications/media/uploads/pubs/533.pdf

The Walt Disney Company (2015). WALL-E - der Letzte räumt die Erde auf: Charaktere. Retrieved 2015, March 31 from http://filme.disney.de/wall-e/charaktere; see also http://pixar.wikia.com/EVE 